Artículo Original

\title{
Relación del perfil salival con el grado de inmunosupresión en pacientes infectados con VIH con y sin tratamiento antirretroviral
}

Salivary profile and its relationship to the degree of immunosuppression in patients with HIV infection with and without highly active antiretroviral treatment

\begin{abstract}
Resumen
El objetivo del estudio fue evaluar el perfil salival de pacientes con infección por VIH con y sin Tratamiento Antirretroviral de Gran Actividad (TARGA), por medio de la evaluación de los síntomas de hipofunción salival (Xerostomía, Disgeusia, Disfagia y Susceptibilidad de desarrollar úlceras orales), la Tasa de Flujo salival no estimulado, el pH salival y la viscosidad salival; estableciendo su relación con el Grado de Inmunosupresión, evaluado por medio del Recuento de Linfocitos CD4/ $\mu l$. Se evaluaron 126 pacientes con infección por VIH con TARGA y sin TARGA que asistieron a la Estrategia Sanitaria de Prevención y Control de ITS, VIH y SIDA del Hospital Nacional Dos de Mayo, Lima-Perú. Los pacientes sin TARGA presentaron porcentajes significativamente mayores de algunos síntomas de hipofunción salival: xerostomía , disgeusia , disfagia y susceptibilidad a desarrollar úlceras orales así como valores significativamente más altos de viscosidad salival, sin embargo, presentaban también valores significativamente más bajos de Tasa de flujo salival no estimulada y de $\mathrm{pH}$ salival respecto a los pacientes con TARGA. Los pacientes con un mayor grado de inmunosupresión presentaron mayores síntomas de hipofunción salival y viscosidad salival así como valores significativamente más bajos de Tasa de Flujo salival no estimulado y de $\mathrm{pH}$ salival respecto a los pacientes con un menor Grado de Inmunosupresión. Se concluyó que existe una alteración del perfil salival de los pacientes con Infección por VIH, la cual es significativamente mayor en los pacientes sin TARGA y en aquellos que tienen un mayor grado de inmunosupresión.
\end{abstract}

\section{Abstract}

The aim of this study was to evaluate the salivary profile of HIV-infected patients with and without Highly Active Antiretroviral Treatment (HAART), evaluating the Symptoms of salivary hypofunction (Xerostomia, Dysgeusia, Dysphagia, and Susceptibility to develop ulcers oral), the Unstimulated Salivary Flow Rate, Salivary $\mathrm{pH}$ and Salivary Viscosity, establishing their relationship with the degree of

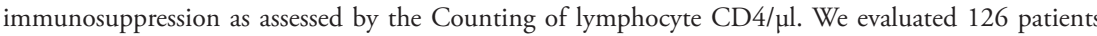
with HIV infection with HAART and without HAART who were attended at the Health Strategy for Prevention and Control of STI, HIV and AIDS at the Hospital Nacional Dos de Mayo, LimaPeru. Patients without HAART had significantly higher rates of some salivary hypofunction Symptoms: Xerostomia, Dysgeusia Dysphagia) and Susceptibility to develop oral ulcers and significantly higher values of Salivary viscosity but values were also significantly lower of Unstimulated Salivary Flow Rate and Salivary $\mathrm{pH}$ compared to patients with HAART. Furthermore, patients with a greater degree of immunosuppression had significantly higher rates of Symptoms of salivary hypofunction and Salivary Viscosit and significantly lower values of Unstimulated Salivary Flow Rate and Salivary pH ,compared to patients with a lower degree of immunosuppression. It was concluded that there was an alteration of the salivary profile of patients with HIV infection, which is significantly higher in patients without HAART and those with a greater degree of immunosuppression.

\section{Introducción}

La saliva es un fluido acuoso hipotónico secretado por las glándulas salivales, de gran importancia para el mantenimiento de la homeostasis de la cavidad oral. ${ }^{1-3}$

Se ha establecido que existe una enfermedad de las glándulas salivales asociada al $\mathrm{VIH},{ }^{4}$ debida a diversas alteraciones estructurales que incluyen lesiones linfoepitaliales, quistes, nodos y otras lesiones que involucran el tejido glandular, ${ }^{5}$ dando lugar a una alteración en la función de las glándulas salivales mayores en el transcurso de la infección por el $\mathrm{VIH},{ }^{6}$ modificando tanto la calidad como la cantidad de la secreción salival. ${ }^{7}$ Todos estos cambios dan lugar a lo que se ha establecido como hipofunción de las glándulas salivales, ${ }^{8}$ asociada a la infección por el VIH, la que provo-

\section{Moisés Vera Cruz ${ }^{1}$ José Cornejo Salazar ${ }^{2}$ Teresa Evaristo Chiyong ${ }^{3}$ Jorge A. Arevalo Abanto ${ }^{4}$ Hugo G. Villanueva Vílchez ${ }^{5}$}

\section{Práctica Privada.}

Dpto. de Cirugía Bucomaxilofacial de la Facultad de Odontología de la UNMSM, Lima-Perú.

Dpto Académico Estomatología Biosocial de la Facultad de Odontología de la UNMSM, Lima-Perú.

4 Coordinador de la Estrategia Sanitaria de Prevención y Control de ITS, VIH y SIDA, del Hospital Nacional Dos de Mayo, Lima-Perú. Profesor responsable de la Cátedra de Fisicoquímica de la Facultad de Farmacia y Bioquímica de UNMSM, Lima-Perú.

Correspondencia:

CD Moisés Vera Cruz

Dirección: Jr. Las adamantinas 2113 ,

Urb. La Huayrona, Distrito de San Juan de Lurigancho, Lima-Perú;

Teléfono: 3870332 / 997622472

E-mail: moiveracruz@hotmail.com

Palabras clave: VIH, síntomas de hipofunción salival, tasa de flujo salival, $\mathrm{pH}$ salival, viscosidad salival, grado de inmunosupresión. Tratamiento antirretroviral de gran actividad.

Keywords: VIH Symptoms of salivary hypofunction, unstimulated Salivary Flow Rate, salivary $\mathrm{pH}$, salivary viscosity, immunosuppression degree and Highly Active Antiretroviral Treatment. caría la consecuente hipofunción de la secreción salival.

Navazesh y col. $2003^{9}$ mencionan como factores de riesgo para el desarrollo de esta hipofunción al recuento de CD4 bajo, así como al uso del Tratamiento Antirretroviral de Gran Actividad (TARGA); sin embargo, Lin y col $2003^{10}$ menciona que las glándulas salivales son afectadas en la fase temprana 
de la infección por el VIH y no necesariamente por la medicación asociada a esta (TARGA).

Basados en los informes anteriormente mencionados y debido a que existe controversia en la literatura en cuanto al momento de la aparición de la hipofunción de las glándulas salivales, así como de la participación del TARGA como un factor de riesgo para el desarrollo de esta alteración; y debido a que de alguna manera esta hipofunción podría coadyuvar al incremento de otras patologías orales muy frecuentes en este tipo de pacientes, se realizó el presente estudio buscando determinar la relación entre el perfil salival y el grado de inmunosupresión de pacientes con infección por VIH, con y sin TARGA;

\section{Material y métodos}

\section{Población estudiada}

Participaron en el estudio 126 pacientes voluntarios con infección por VIH, 65 con y 61 sin TARGA, con una media de $32 \pm 5$ años, que acudían a la Estrategia Sanitaria de Prevención y Control de Infecciones de Transmisión Sexual, VIH y Sida del Servicio de Enfermedades Infecciosas y Tropicales del Hospital Nacional Dos de Mayo, Lima - Perú; atendidos entre los meses de diciembre 2008 y enero 2009 . Se distribuyeron de acuerdo al grado de inmunosupresión ${ }^{11}$ y siguiendo ciertos criterios de inclusión en 3 grupos: Grupo 1: 35 pacientes con una media de $32 \pm 6$ ańos y con más de 500 linfocitos CD4/ $\mu \mathrm{L}$ ), Grupo 2: 50 pacientes con una media de edad de 31 \pm 6 años y con 200 a 500 linfocitos $\mathrm{CD} 4 / \mu \mathrm{L})$, y Grupo 3: 41 pacientes con una media de $32 \pm 5$ ańos y con menos de 200 linfocitos CD $4 / \mu \mathrm{L}$ ).

\section{Recolección de los datos}

Se realizó una entrevista estructurada con el fin de recolectar los datos concernientes a las variables: uso del TARGA y síntomas de hipofunción salival (Xerostomía, Disgeusia, Disfagia y Susceptibilidad a desarrollar úlceras orales).

\section{Colección de la muestras de saliva no estimulada}

Las muestras de saliva fueron colectadas en una sola sesión para cada individuo, bajo las mismas condiciones. Los sujetos estuvieron sentados en una posición de aproximadamente $120^{\circ}$ respecto al piso, con el mínimo de movimientos incluyendo la deglución durante dos minutos, después se enjuagaron la boca con agua purificada y se les pidió que deglutieran el remanente. ${ }^{2,12,13}$

\section{Medición de la tasa de flujo salival}

Se calculó la tasa de flujo de saliva no estimulada mediante el método gravimétrico, colocándose tres rollos de gasa, previamente pesados junto a un frasco de polipropileno de $25 \mathrm{ml}$, uno en el piso de boca a nivel de la desembocadura de los conductos principales submandibular y sublingual y los otros dos en los vestíbulos a nivel de la desembocadura de los conductos principales parotídeos; luego de esperar cinco minutos, las gasas fueron removidas y colocadas en frascos antes mencionados y vueltos a pesar, hallándose la diferencia y dividiéndola entre los cinco minutos, obteniéndose valores en $\mathrm{ml} / \mathrm{min}$. (Considerando que 1 $\mathrm{g} \approx 1 \mathrm{ml}){ }^{2,12,13}$ Los frascos con las gasas $\mathrm{y}$ las muestras fueron pesados en una balanza analítica digital (EPSO5-Kinlee) de $0.01 \mathrm{~g}$ de precisión, con una capacidad máxima de 100 gr; descartándose las muestras que contenían sangre, lápiz labial o algún otro detrito.

\section{Medición del pH salival}

Se solicitó al paciente que siguiera recolectando saliva no estimulada durante algunos minutos, mediante la Técnica de expectoración, ${ }^{13}$ hasta obtener aproximadamente $6 \mathrm{ml}$, procediéndose a tomar la medida del $\mathrm{pH}$, por medio de la cinta universal de $\mathrm{pH}^{14}(\mathrm{pH}$ Test Paper Special, Guangzhou Sinosource Trade Co., Ltd.-China, () Gold Supplier).

Cuadro 1. Perfil salival según el uso del TARGA; de los pacientes VIH+ que acuden al Servicio de Enfermedades infecciosas y tropicales (SEIT) del Hospital Nacional Dos de Mayo.

\begin{tabular}{|c|c|c|c|c|c|}
\hline \multirow{3}{*}{ VARIABLES } & & \multicolumn{4}{|c|}{ Uso del TARGA } \\
\hline & & \multicolumn{2}{|c|}{ Sin TARGA } & \multicolumn{2}{|c|}{ Con TARGA } \\
\hline & & No & $\%$ & No & $\%$ \\
\hline \multirow{7}{*}{ 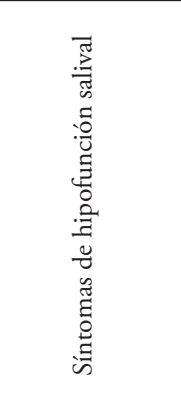 } & No presenta & 36 & 59,0 & 59 & 90,8 \\
\hline & Sí presenta & 25 & 41,0 & 6 & 9,2 \\
\hline & No presenta & 35 & 57,4 & 48 & 73,8 \\
\hline & Sí presenta & 26 & 42,6 & 17 & 26,2 \\
\hline & No presenta & 46 & 75,4 & 62 & 95,4 \\
\hline & Sí presenta & 15 & 24,6 & 3 & 4,6 \\
\hline & No presenta & 34 & 55,7 & 57 & 87,7 \\
\hline a úlceras orales & Sí presenta & 27 & 44,3 & 8 & 12,3 \\
\hline \multirow{4}{*}{ Nivel de la Tasa de Flujo Salival } & Bajo & 25 & 41,0 & 5 & 7,7 \\
\hline & Disminuido & 15 & 24,6 & 17 & 26,2 \\
\hline & Normal & 21 & 34,4 & 43 & 66,2 \\
\hline & Ácido & 36 & 59,0 & 14 & 21,5 \\
\hline \multirow[t]{2}{*}{$\mathrm{pH}$ salival } & Neutro & 15 & 24,6 & 28 & 43,1 \\
\hline & Básico & 10 & 16,4 & 23 & 35,4 \\
\hline \multirow{3}{*}{ Grado de viscosidad salival } & Bajo & 2 & 3,3 & 14 & 21,5 \\
\hline & Normal & 24 & 39,3 & 34 & 52,3 \\
\hline & Alto & 35 & 57,4 & 17 & 26,2 \\
\hline
\end{tabular}

\section{Medición de la viscosidad salival}

Se calculó la viscosidad de la saliva no estimulada mediante el Método de Ostwald, ${ }^{14-15}$ utilizándose el Viscosímetro de Ostwald (CF VISKOSIMETER, SCHOTT \& GEN, MAINZ, JENA"GLAS; App. Nr. 30993; $\mathrm{K}=0,008484$ ), en el Laboratorio de Fisicoquímica de la Facultad de Farmacia y Bioquímica de la Universidad Nacional Mayor de San Marcos, Lima-Perú. Para lo cual se midieron $5 \mathrm{ml}$ de la muestra y se colocaron por la rama ancha del viscosímetro; luego se aspiró la muestra por la rama angosta del viscosímetro con la ayuda de una jeringa hipodérmica y una manguera de goma acoplada a su punta, llenando la primera ampolla y superando la línea superior marcada del viscosímetro; luego se le colocó en un vaso pirex con agua a temperatura constante de $20{ }^{\circ} \mathrm{C}$ durante cinco minutos y se dejó escurrir la muestra, tomándose tiempo que le demoró en fluir desde la línea superior hasta la línea inferior de la ampolla, procedimiento que se realizó tres veces obteniéndose un promedio. Se realizó lo mismo con $5 \mathrm{ml}$ de agua destilada y tomando en cuenta que la densidad del agua a $20^{\circ} \mathrm{C}$ es de 1,002 cP (Centipoise), la viscosidad de la muestra en cP se obtuvo de la división del tiempo que le demoró a la muestra de saliva entre el tiempo que le demoró al agua en fluir entre la marcas del Viscosímetro. ${ }^{14,15}$ 


\section{Resultados}

La presencia de xerostomía se encontró en el 24,6\% de la población en estudio, apreciándose un porcentaje significativamente más alto en los pacientes sin TARGA (41\%) respecto a los pacientes con TARGA $(9,2 \%)(\mathrm{p}=0,00)$. La Disgeusia se presentó en el 34,1\%; encontrándose que los pacientes sin TARGA $(42,6 \%)$ presentaron un porcentaje más alto que los pacientes con TARGA $(26,2 \%)$, no evidenciándose una diferencia significativa $(p=, 051)$ entre ambos grupos. La disfagia se presentó en un $14,3 \%$ encontrándose un porcentaje significativamente más alto que $(\mathrm{p}=, 001)$ en los pacientes sin TARGA

Cuadro 2. Tasa de flujo, $\mathrm{pH}$ y viscosidad salival en relación con el grado de inmunosupresión.

\begin{tabular}{|c|c|c|c|c|c|}
\hline & \multirow[b]{2}{*}{ Uso del TARGA } & \multicolumn{3}{|c|}{ GRADO DE INMUNOSUPRESIÓN } & \multirow{2}{*}{$\begin{array}{c}\text { ANOVA } \\
\mathbf{P}\end{array}$} \\
\hline & & Grado 1 & Grado 2 & Grado 3 & \\
\hline \multirow{3}{*}{$\begin{array}{c}\text { Tasa de flujo } \\
\text { salival }\end{array}$} & Sin TARGA & $0,553 \pm, 227$ & $0,267 \pm, 063$ & $0,120 \pm, 039$ &, 000 \\
\hline & Con TARGA & $0,748 \pm, 285$ & $0,422 \pm, 161$ & $0,247 \pm, 093$ &, 000 \\
\hline & Total & $0,659 \pm, 274$ & $0,363 \pm, 152$ & $0,167 \pm, 088$ &, 000 \\
\hline \multirow{3}{*}{ pH salival } & Sin TARGA & $7,406 \pm, 554$ & $6,526 \pm, 589$ & $5,788 \pm, 493$ &, 000 \\
\hline & Con TARGA & $7,711 \pm, 535$ & $6,952 \pm, 506$ & $6,400 \pm, 660$ &, 000 \\
\hline & Total & $7,571 \pm, 558$ & $6,790 \pm, 572$ & $6,012 \pm, 627$ &, 000 \\
\hline \multirow{3}{*}{$\begin{array}{l}\text { Viscosidad } \\
\text { salival }\end{array}$} & Sin TARGA & $1,471 \pm, 039$ & $1,504 \pm, 029$ & $1,600 \pm, 066$ &, 000 \\
\hline & Con TARGA & $1,395 \pm, 049$ & $1,487 \pm, 073$ & $1,516 \pm, 031$ &, 000 \\
\hline & Total & $1,430 \pm, 059$ & $1,493 \pm, 060$ & $1,569 \pm, 069$ &, 000 \\
\hline
\end{tabular}

Grado 1= >500 linfocitos CD4/ $\mu \mathrm{L}$; Grado 2= 200-500 linfocitos CD4/ $\mathrm{LL}$; Grado $3=(<200$ linfocitos $\mathrm{CD} 4 / \mu \mathrm{L}$

\section{Discusión}

Schiødt $\mathrm{M}^{5}$ menciona que la hipofunción de las glándulas salivales se define con la presencia de xerostomía y/o inflamación de las glándulas salivales mayores, al respecto menciona que esta alteración está asociada a cuentas de T4 bajas y al desarrollo del estadio SIDA; ${ }^{4}$ estableciéndose, como lo menciona Navazesh y col., ${ }^{9}$ como factores de riesgo el Recuento de CD4 bajo y el uso del TARGA; sin embargo, Lin y col. ${ }^{10}$ mencionan que la función de las glándulas salivales es afectada en la fase temprana de la infección por el VIH y no necesariamente por la medicación xerogénica. En la presente investigación la hipofunción de las glándulas salivales se ve evidenciada en los resultados obtenidos; encontrándose que de acuerdo a una progresiva disminución del recuento de linfocitos CD4, evidenciada por el grado de inmunosupresión, tanto los síntomas de hipofunción salival, así como el valor del grado de viscosidad de la saliva no estimulada, van en au-

$(24,6 \%)$ respecto a los pacientes con TARGA (4,6 \%). La susceptibilidad de desarrollar úlceras orales se presentó en un $27,8 \%$ encontrándose un porcentaje significativamente más alto $(\mathrm{p}=, 000)$ en el grupo sin TARGA $(44,3 \%)$ respecto a con TARGA $(12,3 \%)$

Con respecto a la Tasa de Flujo Salival no Estimulado, el pH salival y la viscosidad salival, se encontraron diferencias significativas $(\mathrm{p}=.000)$ entre los pacientes sin TARGA respecto a los pacientes con TARGA. Se evidenció también que a mayor grado de inmunosupresión todos estos síntomas y signos se presentaron con mayor frecuencia, siendo estas diferencias estadísticamente significativas. min) y el estar infectado por el VIH; así como Lin y col., ${ }^{10}$ quien estableció una diferencia significativa entre la tasa una diferencia significativa entre la tasa
de flujo salival de los pacientes VIH+ y VIH.

Asimismo, se han encontrado valores significativamente más bajos en los pacientes sin TARGA respecto a los pacientes con TARGA, lo que difiere de lo encontrado por Lin y col $2003,{ }^{10}$ el cual encontró que no existía diferencia significativa entre ambos grupos. Los pacientes con un mayor grado de inmunosupresión presentaron medias significativamente más bajas de Tasa de flujo salival no estimulado.

Los valores de $\mathrm{pH}$ salival encontrados, no difieren de los hallados por Fenoll y col. ${ }^{3}$ $(6,7903 \pm 0,2874)$, pero que son inferiores a los establecidos por Jiménez $(7,2)$ y Rockenbach y col ${ }^{19}(7,5 \pm 0,4)$, en poblaciones sanas, sin factores de riesgo.

Coates y col. ${ }^{7}$ mencionan que los pacientes con infección por VIH en un $46 \%$ poseían un nivel de $\mathrm{pH}$ salival ácido $(<6,5)$, en la presente investigación se pudo determinar que los pacientes sin TARGA, además de los pacientes en el grupo grado 3 de inmunosupresión tienen medias menores a 6,5. Por otro lado, los pacientes con un mayor grado de inmunosupresión presentaron medias significativamente más bajas de $\mathrm{pH}$ salival.

En la presente investigación, los pacientes sin TARGA y con grado 3 de inmunosupresión, presentaron medias superiores a las registradas por Ortega y col ${ }^{15}$ $(1,49 \pm 0,49)$ y Jiménez ${ }^{14}(1,48 \mathrm{cP})$, en poblaciones sanas, sin factores de riesgo.

\section{Conclusión}

Los pacientes sin TARGA presentaron un porcentaje significativamente mayor 
de xerostomía, disgeusia, disfagia y susceptibilidad de desarrollar úlceras orales que los pacientes con TARGA siendo estos síntomas significativamente más altos en los pacientes con un mayor grado de inmunosupresión.

Valores significativamente más bajos de Tasa de flujo salival no estimulado, $\mathrm{pH}$ salival se observaron en los pacientes sin TARGA.

\section{Agradecimientos}

Al M.C. Jorge A. Arévalo Abanto, Coordinador de la Estrategia Sanitaria de Prevención y Control de ITS, VIH y SIDA del Hospital Nacional Dos de Mayo, Lima-Perú; por su colaboración en el planeamiento y ejecución del estudio.

Al Mg. Q.F. Hugo G. Villanueva Vílchez, profesor responsable de la cátedra de Fisicoquímica de la Facultad de Farmacia y Bioquímica de UNMSM, por su colaboración con el análisis de las muestras.

\section{Referencias bibliográficas}

1. Bascones A, Tenovuo J, Ship J, Turner M, Mac-veigh I, y col. Conclusiones del Simposium 2007 de la Sociedad Española de Medicina Oral sobre Xerostomía. Síndrome de Boca Seca. Boca Ardiente. Avances en Odontoestomatología. 2007; 23 (3): 119-126.

2. Banderas J, González M, Sánchez M, Millan E, López A, Vilchis A. Flujo y concentración de proteínas en saliva total humana. Salud pública de México. 1997; 39(5).

3. Fenoll C, Muñoz J, Sanchiz V, Herreros B, Hernández V, Mínguez M, y col. Unstimulated salivary flow rate, $\mathrm{pH}$, and buffer capacity of saliva in healthy volunteers. Revista Española de Enfermedades Digestivas. 2004;96 (11): 773-783
4. Schiødt M, Greenspan D, Dodd C, Chernoff D, Wara D, Leggott P y col. Natural history of HIVassociated salivary gland disease. Revista International Conference on AIDS. 1989; 49(5): 473

5. Schiødt M. HIV-associated salivary gland disease: a review. Oral Surg Oral Med Oral Pathol. 1992; 73(2): 164-167.

6. Atkinson J, Yeh C, Bermudez D, Fox P, Baum B. Longitudinal evaluation of major salivary gland function in HIV-1 infected patients. Journal of Oral Pathology \& Medicine. 1989; 18 (8): 469470 .

7. Coates E, Wilson D, Logan R. The effects of HIV infection on the quality and flow of saliva. Revista International Conference on AIDS. 1998; 12: 1015-1016.

8. Navazesh M, Mulligan R, Komaroff E, Redford M, Greenspan D, Phelan J.The Prevalence of Xerostomía and Salivary Gland Hypofunction in a Cohort of HIV-positive and At-risk Women. J Dent Res, 2000; 79(7): 1502-1507.

9. Navazesh M, Mulligan R, Barrón Y, Redford M, Greenspan D, Alves M, Phelan J. A 4-year longitudinal evaluation of xerostomia and salivary gland hypofunction in the Women's Interagency HIV Study participants. Oral Surg Oral Med Oral Pathol Oral Radiol. 2003; 95(6): 693-698.

10. Lin L, Johnson A, Stephan T, Yeh K. Alteration in Salivary Function in Early HIV Infection. J Dent Res. 2003; 82(9):719-724.

11. Centers for Disease Control. Revised Classification system for HIV Infection and expanded surveillance case definition for AIDS among adolescents and adults. Revista MMWR. 1993; 41(17).
12. Najat F. Signs of oral dryness in relation to salivary flow rate, $\mathrm{pH}$, buffering capacity and dry mouth complaints. Revista BMC Oral Health. 2007; 7: 15.

13. Navazesh M, Kumar S. Measuring salivary flow. Challenges and opportunities. J Am Dent Assoc. 2008; 139 (5) ): 35S-40S.

14. Jiménez R. Importancia del pH, flujo y viscosidad salival sobre el desarrollo de caries dental en mujeres gestantes del primer trimestre. [Tesis de bachiller]. Universidad Nacional Mayor de San Marcos. Lima - Perú, 2004.

15. Ortega M, Calzado M, Pérez M. Evaluación del flujo y viscosidad salival y su relación con el índice de carie. Medisan. 1998; 2(2): 33-39.

16. Sherson W, Taylor B. Xerostomía in an HIV positive cohort. Revista Annu Conf Australas Soc HIV Med. 1997; 13-16(9): 132

17. Younai F, Marcus M, Freed J, Coulter I, Cunningham W, Der C, y col. Self-reported oral dryness and HIV disease in a national sample of patients receiving medical care. Oral Surg Oral Med Oral Pathol Oral Radiol Endod. EEUU. 2001; 92 (6): 629-636.

18. Salas J, Küstner C, López L, Roselló L, Ocaña R. Efecto de los tratamientos antirretrovirales en las manifestaciones orales de los pacientes VIH+. Avances en odontoestomatología. 2006; 22(6): 315-326

19. Rockenbach M, Marinho S, Veeck E, Lindemann L, Shinkai R. La proporción de flujo salival, $\mathrm{pH}, \mathrm{y}$ concentraciones de calcio, fosfato y sIgA en las mujeres embarazadas y non-embarazadas. Head \& Face Medicine. 2006; Vol 2: 44.

Fecha de recepción: 22 de junio 2009

Fecha de aprobación: 05 de enero 2010 